\title{
A shock harness for rats: Resistance as a function of shock intensity'
}

\author{
NANCY K. MELLO AND JACK H. MENDELSON \\ MASSACHUSETTS GENERAL HOSPITAL AND HARVARD MEDICAL SCHOOL
}

\begin{abstract}
Abstraet
The efficiency of a harness device for administering consistent electric shocks to rats was evaluated by measuring changes in resistance as a function of shock intensity, both within a single shock and over the course of 15 days. Resistance within a single session was most constant at the highest shock level used (4.25 ma) and did not increase significantly as a function of time at any of the four shock intensities employed.
\end{abstract}

\section{Introduction}

The several problems associated with presentation of shock through an electrified grid floor (England, 1964) have lead to the development and increasingly successful use of implanted electrodes in various species (Azrin, 1959; Silver et al, 1964). We have found that a nonavoidable and consistent shock can also be delivered to rats through a small metal harness which can be attached before each conditioning session.

The efficiency of the shock harness device has been evaluated by examining changes in a rats resistance: (a) within a single shock of $0.6 \mathrm{sec}$.duration; (b) during a single session in which 100 shocks were administered and (c) over the course of 15 daily sessions. Each of these three indices was studied as a function of four levels of shock ranging from 1.5 to $4.25 \mathrm{ma}$.

Resistance was determined from the slope of a voltage versus current curve, photographed from an oscilloscope display. The method described is equally applicable to resistance measurements with implanted electrodes and other types of harness electrodes.

\section{Method}

The shock harness consists of a flexible, stainless steel pipe clamp (45 gm in weight) which can be adjusted to the girth of the rat. A semi-circle of copper $(1 / 2$ in $\times 1-1 / 2$ in $\times 0.02$ in) is attached to and insulated from the inside of the steel band. Input leads, encased in flexible metal shielding, are attached to both the steel band and the copper strip, and joined to a connector on the roof of the experimental chamber.

In order to determine the extent to which movement or possible tissue changes would alter resistance in this harness, four naive hooded rats, weighing 300-350 gm, were given 100 shocks of $0.6 \mathrm{sec}$. duration, at 20 sec. intervals, every day for 15 days. The shock source delivers 60 cycle AC current to the rat through $10,000 \mathrm{ohm}$ series resistor. Four shock intensities, approximately $0.5,1.5,3$, and 4.25 milliamperes (ma) were used. Each rat received only one shock level throughout the experiment.

Following Ohms law, it is possible to calculate the resistance of the rat during a single shock, by plotting voltage (V) against current (I) on an oscilloscope (Tektronix 502). A typical V I trace is shown in Fig. 1a. Similarly, both voltage changes (upper trace) and current changes (lower trace) can be plotted as a function of time within a single shock (Fig, 1b)。

To calculate the instantaneous resistance of an animal from an oscilloscope tracing of a V I curve, it is first necessary to determine the vertical and horizontal extent of the V I curve in centimeters. The vertical excursion, voltage (V) is then divided by the horizontal excursion current (I) and the resulting quotient is multiplied by a constant slope factor (C) to yield a resistance value $(R)$ :

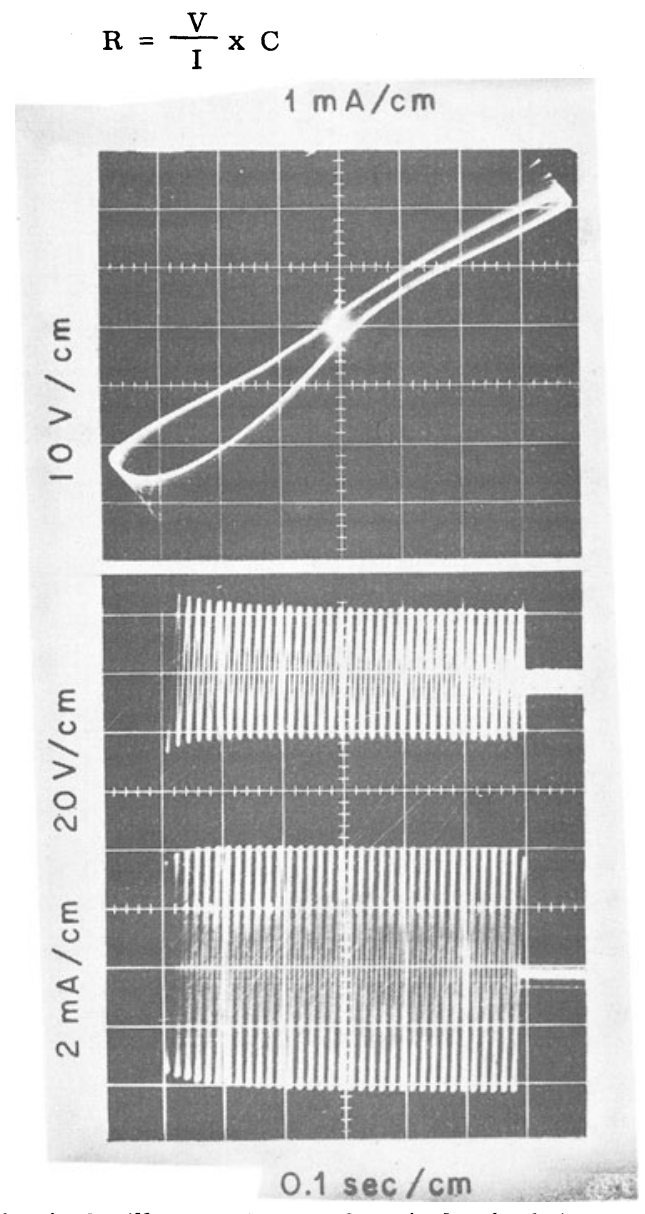

Fig. 1. Oscilloscope traces of a single shock (approxımately $3 \mathrm{ma}$ ) delivered to a rat through a shock harness. (a) Voltage (V) is plotted against current (I) during a single shock. The resistance can be calculated from the slope of the $V$ I curve. (b) Voltage (V) and Current (I) are plotted against time during a single shock. 
The appropriate slope factor (C) is computed from the calibrations of the vertical and horizontal amplifiers on the oscilloscope. The ordinate is divided by the abscissa and the quotient is the slope factor (C). For example, in Fig. 1a:

$$
\begin{aligned}
& \frac{10 \mathrm{v} / \mathrm{cm}}{1 \mathrm{ma} / \mathrm{cm}}=10 \text { slope factor }(\mathrm{C}) \\
& \mathrm{R}=\frac{4.6}{7.6} \times 10 \text { or } \mathrm{R}=6.05 \text { kilohms }
\end{aligned}
$$

During each of the first five sessions, V I curves were photographed for shocks 1-6, 45-50, and 95-100. Each rat's resistance for these three groups of shocks was computed from the slope of the V I curves and the mean resistance was determined. Each session lasted for $34 \mathrm{~min}$. In order to examine possible long term effects of shock, 10 additional daily sessions of 100 shocks were run. V I curves were photographed during sessions 10 and 15 .

\section{Results and Diseussion}

The mean resistance values within each session for sessions 1-6, 10 and 15 are shown in Fig. 2. The range is shown only when it is large enough to plot. Resistance values for the rat receiving $0.5 \mathrm{ma}$ shocks are not presented since the slopes of the V I curves obtained were too steep to be read accurately. Resistance appeared to be between 100 and 200 kilohms, but no finer judgment could be made from the oscilloscope traces. Comparable data was obtained on a second rat shocked at $0.5 \mathrm{ma}$.

It appears that the relative constancy of resistance varies in proportion to the shock level, since resistance with in a single session is most constant at the highest shock level (4.25 ma). At each of the lower shock levels, resistance tends to be highest at the beginning of each session and decreases during the course of a session. These data indicate that there was an effective current increase within a single session. The drop in resistance within a single session was most marked at the lowest shock level (1.5 ma).

However, resistance variability between sessions is greatest at the higher shock levels. The absence of a distinct upwards trend suggests that no significant tissue changes occurred as a function of prolonged exposure to shocks as high as $4.25 \mathrm{ma}$. At the lowest level, there is a marked decrease in resistance between sessions. Careful studies of resistance changes as a function of time (Ferraro et al, 1964) in rats shocked through implanted electrodes, have shown a gradual increase in resistance through time even though the shock intensity used did not exceed $.3 \mathrm{ma}$. The total range of resistance values obtained using the shock harness (2 to 14 kilohms) was comparable to values reported by Silver et al (1964), (using implanted

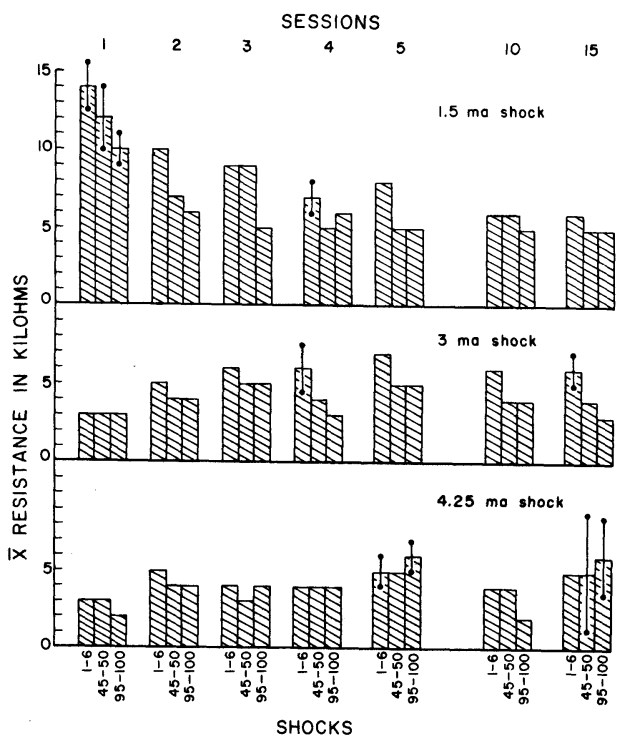

Fig. 2. Mean resistance values for three rats, shocked at three intensities through a harness device. Resistance, calculated from $V$ I curves is shown as a function of time.

electrodes) at similar shock intensities in the descending series.

The plot of voltage and current changes against time (Fig. 1b) for a single shock shows that resistance stabilizes within less than $0.2 \mathrm{sec}$. This suggests that shock durations should not be less than $0.2 \mathrm{sec}$. for constant efficacy. Since the typical V I curve shown in Fig. 1a is not simply a straight line, this indicates that the rat is not a simple resistor. Rather, the rat is both resistive and capacitive and within a single shock, resistance decreases and then stabilizes. In the example shown, the change is from a slope of approximately 1 to a slope of about 0.5. A similar conclusion was reached by Silver et al (1964) using a different method of impedance measurement with implanted electrodes.

\section{References}

Azrin, N. A technique for delivering shock to pigeons. J. exp. Anal. Behav., 19959, 2, 161-163.

England, S. J. M. A constant power shock source and electronic grid scrambler with an historical introduction. Percept. mot. Skills, 1964, 18, 961-975.

Ferraro, D. P., Silver, M. P., \& Snapper, A. G. Impedance-time functions in the white rat with chronic body electrode implants. Psychon. Sci., 1964, 1, 393-394.

Silver, M. P., Schoenfeld, W. N., Snapper, A. G., \& Locke, B. Impedance-voltage functions in the white rat with chronic body electrode implants. Psychon. Sci., 1964, 1, 61-62.

\section{Note}

1. This work was supported by a USPH Grant (M-4814) from the National Institute of Mental Health. We are grateful to R. B. Parente of the Electrical Engineering Department, Massachusetts Institute of Technology for consultation. We thank R. R. Ballard for his excellent technical assistance. 